\title{
Prosodic Structure of Turkish and Hindi: A Comparative Analysis
}

\author{
Hazel Zeynep KURADA \\ Hacettepe University
}

\begin{abstract}
Universally, the functioning of each language system is composed of some specific sequences. As one of these regulations, prosodic structure, a phonological component, includes supra-aural facts above segmental phonemes. Although the suprasegmental phonemes appear in every language, they may not exhibit a contrastive relationship in each language as a result of varying structures of the languages. For instance, word stress is weak and does not function as a meaning distinctive in some languages (French, Hungarian, Finnish etc), whereas in some languages (Turkish, Italian, English etc.) word stress is highly strong and functions as a meaning distinctive. To point out such discrepancies is crucial to prevent intelligibility problems during communication process as such discriminations between languages mostly cause speech intelligibility problems among foreign language speakers. In this theoretical research, for the first time in linguistics literature, suprasegmental structures like word stress, duration, juncture, intonation and focus phonemes have been discussed comparatively in Hindi and Turkish. It has been observed that the functioning of suprasegmental phonemes as a prosodeme is quite different between Hindi and Turkish as both languages are basically differentiate on account of length, nasalisation and aspiration of segmental phonemes. Within the framework of observed regulations, major discrepancies which may lead a speech intelligibility problem among speakers have been pointed out and also, possible reasons of such discrepancies in both languages are deeply discussed.
\end{abstract}

Keywords: intelligibility; phoneme; prosodeme; segmental; suprasegmenta 